\title{
Assessment of Radioactive Iodine Treatment in Hyperthyroidism Sudanese Patients
}

\author{
Mahdi Ahmed Ibrahim ${ }^{1,2}$, M. E. M. Garelnabi ${ }^{1}$, Abdoelrahman Hassan A. B ${ }^{3}$ \\ ${ }^{1}$ Sudan University of Science and Technology, COMRS, Khartoum, Sudan \\ Nuclear Medicine Department, Radiation and Isotopes Center of Khartoum \\ ${ }^{2}$ Sudan University of Science and Technology, COMRS, Khartoum, Sudan \\ ${ }^{3}$ Sudan University of Science and Technology, COMRS, Khartoum, Sudan \\ Anatalya Diagnostic Medical Center, Khartoum, Sudan
}

\begin{abstract}
Hyperthyroidism is considered as one of common pathological problem associated with thyroid gland, in which thyroid hormone level were increased. This a prospective study attended to assess the role of thyroid ablation using I-131 in treatment of hyperthyroidism in Sudanese population, A 120 patient were treated by using of $I^{131}$ at radiation and isotopes center of Khartoum in

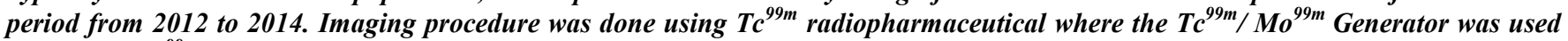
to produce Tc ${ }^{99 m}$, dose caliber and Dual head Gama camera was used to scan these patient. The level of thyroid hormone were recorded before, and after 3, 6, 9, and 12 month after treatment in order to assess the decrement level of these hormone and therefore performing a quantitative assessment of hyperthyroidism treatment, the result showed that the mean value of these hormones was 6.9, 4.2, 2.8, and 2.5 (nmol/L) for T3, 222.1, 134, 119, 119.2 and 113.4 (nmol/L) for T4, and 0.37, 6.34, 5.3, 2.8, and 2.3 (nmol/L) for TSH respectively. Pair sample two tailed test was performed; a significant difference between the thyroid hormone level was noted before the RAI treatment and after 3, 6, 9, and 12 month later after the treatment, at Confidence level of $(C L=95 \%)$ and ( $p$ value= 0.05$)$, the test were significant at $P=0.000$. Causative disease of hyperthyroid function found to be due to Graves' disease in 45 patient (54\%) of the collected samples, 28 (23.3\%) by TMNG, 30 (25\%) due to Diffuse toxic goiter and $6.7 \%$ related to the autonomous toxic nodules. This study concludes the hyperthyroidism treatment using $I^{131}$ considered effective and cause significant difference in thyroid hormone level after treatment.
\end{abstract}

Keywords: Thyroid gland, $\mathrm{I}^{131}$, Hyperthyroidism and Thyroid hormone

\section{Introduction}

The thyroid is made up of the isthmus which overlying the 2nd and 3rd rings of the trachea, the lateral lobes which each extending from the side of the thyroid cartilage downwards to the 6th tracheal ring and an inconstant pyramidal lobe which projecting upwards from the isthmus, usually on the left side, which represents a remnant of the embryological descent of the thyroid. The thyroid gland is enclosed in the pretracheal fascia, covered by the strap muscles and overlapped by the sternocleidomastoids. The anterior jugular veins course over the isthmus. When the thyroid enlarges, the strap muscles stretch and adhere to the gland so that, at operation, they often appear to be thin layers of fascia. On the deep aspect of the thyroid lie the larynx and trachea, with the pharynx and oesophagus behind and the carotid sheath on either side. Two nerves lie in close relationship to the gland; in the groove between the trachea and oesophagus lies the recurrent laryngeal nerve and deep to the upper pole lies the external branch of the superior laryngeal nerve passing to the cricothyroid muscle [1-4].

Nuclear Medicine is the section of science that utilizes the properties of radiopharmaceuticals in order to derive clinical information of the human physiology and biochemistry. The radiopharmaceutical follows its physiological pathway and it is concentrated on specific organs and tissues for short periods of time. Then, the patient is positioned under a nuclear medicine equipment which can detect the radiation emitted by the human body resulting in images of the biodistribution of the radiopharmaceutical. In Nuclear Medicine, there are two main methods of patient imaging, the imaging with Planar Imaging, Dynamic Imaging or SPECT and the PET. During the last decade, hybrid systems have been developed integrating the $\mathrm{CT}$ technique with either SPECT or PET resulting in SPECT/CT and PET/CT respectively. This section will concentrate on study of the effect of thyroid disease in thyroid hrmone concentration and levels by using of planar imaging, SPECT and SPECT/CT methods [5-7]. The gamma camera is composed of a collimator, a scintillator crystal usually made of $\mathrm{NaI}$ (or CsI), the photomultiplier tubes, the electronic circuits and a computer equipped with the suitable software to depict the nuclear medicine examinations. In planar imaging, the patient, having being delivered with the suitable radiopharmaceutical, is sited under the gamma camera head. The gamma camera head remains stable at a fixed position over the patient for a certain period of time, acquiring counts (disintegrations). These will constitute the radiopharmaceutical distribution image. The counts measured in a specific planar projection originate from the whole thickness of patient [8-9]. In SPECT, the gamma camera head rotates around the patient remaining at well-defined angles and acquiring counts for specific periods of time per angle. What makes SPECT a valuable tool in nuclear medicine is the fact that information in the three dimensions of the patient can be collected in a number of slices with a finite known volume (in voxels).Thus SPECT technique is used to display the radiopharmaceutical distribution in a single slice removing the contribution from the overlying and

\section{Volume 4 Issue 12, December 2015}




\section{International Journal of Science and Research (IJSR) \\ ISSN (Online): 2319-7064 \\ Index Copernicus Value (2013): 6.14 | Impact Factor (2013): 4.438}

underlying tissues. In order to obtain the most accurate quantitative data from SPECT images, two issues that have to be resolved are the attenuation correction and the Compton scattering that the photons are undergone until reach and interact with the slice of interest tissues. As an examining organ has certain dimensions, each slice along the axis of the gamma camera has different distance from the detector. Thus, each photon experiences different attenuation. These two phenomena usually lead to distortion of the measured activity concentration [10-11].

Hyperthyroidism refers to any condition in which the body has too much thyroid hormone or overactive tissue within the thyroid gland, resulting in overproduction and thus an excess of circulating free thyroid hormones: thyroxine $\left(\mathrm{T}_{4}\right)$, triiodothyronine $\left(\mathrm{T}_{3}\right)$, or both. Symptoms may include weight loss, nervousness, irritability, increased perspiration, a racing heart, hand tremors, anxiety, difficulty sleeping, increased bowel movements, fine brittle hair, and muscular weakness especially in the upper arms and thighs. In Graves' disease, a bulging of one or both eyes may occur. A classification of hyperthyroidism causes had been made by William et.al 2005 , in which he stated that $95 \%$ associated with increased radioiodine uptake in patient with Graves' and Plummer's disease. Many treatment options had been proposed since 1941 and have since evolved to the treatment modality of choice for the majority of adult patients, particularly in the USA. The effectiveness of radioiodine treatment for hyperthyroidism is due to radiation-induced cellular damage resulting from high-energy beta emission, the magnitude of which is directly proportional to the radiation dose received by the thyroid gland.

This paper aimed to discuss the role of thyroid treatment of hyperthyroidism by using of $\mathrm{I}^{131}$ radioisotopes in Sudanese population.

\section{Material and method}

A cross-sectional (descriptive) hospital based prospective study carried out to evaluate and to assess the role of $\mathrm{I}^{131}$ treatment of hyperthyroidism, patient referred to the thyroid clinic in nuclear department, radiation and isotopes center of Khartoum, C/O enlarged thyroid gland with thyroid hormone disturbance; physician advice the patient to perform laboratory investigation, and thoracic inlet x-ray used to evaluate the hormone level and the airway passage, thyroid function test performed using $5 \mathrm{ml}$ of blood, test tube, centrifuge to separate the plasma from the blood component, I125, antibodies, and Gamma counter (PC-RIA.MAS STRATEC, Germany), An elevated RAIU confirms that endogenous thyroidal secretion is the source of the hyperthyroidism and aids in excluding other etiologies of hyperthyroidism, such as silent thyroiditis, sub-acute thyroiditis, postpartum thyroiditis, iodine-induced hyperthyroidism, and factitious hyperthyroidism, all of which are associated with a low RAIU. Tc $99 \mathrm{~m} / \mathrm{Mo} 99 \mathrm{~m}$ generator, Dual head gamma Camera $\left(\mathrm{FOV}=53 \mathrm{X} 49 \mathrm{~cm} 2\right.$, Nucline ${ }^{\mathrm{TM}}$ SPIRT DH-V, MEDISO-Company, Hungary) SPECT imaging was used to scan the patient to assess cause of hyperthyroidism and the thyroid uptake was recorded, the level of thyroid hormone was recorded before starting radioiodine treatment and 3, 6, 9, 12 months after treatment in order to assess the level of thyroid hormone in these periods and therefore assessing the treatment response and the outcome.

\section{Result}

Table 1: Demonstrate mean \pm STD of thyroid hormone for 120 patients having mean age of $42.98 \mathrm{yrs}$.

\begin{tabular}{|c|c|c|c|}
\hline Period & $T 3$ & $T 4$ & $T S H$ \\
\hline Before Tx & $6.9 \pm 6.3$ & $222.1 \pm 110.47$ & $0.37 \pm 0.7$ \\
\hline After 3m & $4.2 \pm 7.9$ & $134 \pm 88.4$ & $6.34 \pm 15.5$ \\
\hline After 6m & $2.8 \pm 3.5$ & $119 \pm 66.7$ & $5.3 \pm 13.1$ \\
\hline After 9m & $2.8 \pm 2.9$ & $119.2 \pm 59.5$ & $2.8 \pm 4.1$ \\
\hline After $12 \mathrm{~m}$ & $2.5 \pm 1.8$ & $113.36 \pm 47.2$ & $2.3 \pm 2.7$ \\
\hline
\end{tabular}

Table 2: showed the age group for collected samples

\begin{tabular}{|c|c|c|}
\hline Age group & Frequency & Percent (\%) \\
\hline $10-19$ & 3 & 2.5 \\
\hline $20-29$ & 16 & 13.3 \\
\hline $30-39$ & 24 & 20.0 \\
\hline $40-49$ & 33 & 27.5 \\
\hline $50-59$ & 32 & 26.7 \\
\hline $60-69$ & 10 & 8.3 \\
\hline $70-79$ & 2 & 1.7 \\
\hline Total & 120 & 100.0 \\
\hline
\end{tabular}

Table 3: Showed the frequancy distribution of the most causes of thyroid hyperthyrodism.

\begin{tabular}{|c|c|c|}
\hline Variables & Frequency & Percent \\
\hline Graves' & 54 & 45.0 \\
\hline TMNG & 28 & 23.3 \\
\hline Diffuse toxic goiter & 30 & 25.0 \\
\hline Autonomous toxic nodule & 8 & 6.7 \\
\hline Total & 120 & 100.0 \\
\hline
\end{tabular}

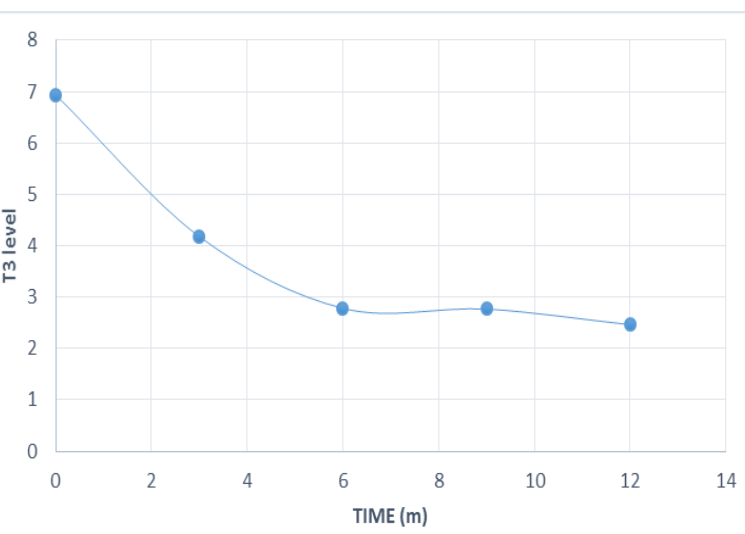

Figure 1: line graph showed the decrement of T3 level with time a value $(0)$ represent pretreatment time at $\mathrm{x}$-axis.

$y=-0.3455 x+5.8996, R^{2}=0.7736$ 


\section{International Journal of Science and Research (IJSR) \\ ISSN (Online): 2319-7064}

Index Copernicus Value (2013): 6.14 | Impact Factor (2013): 4.438

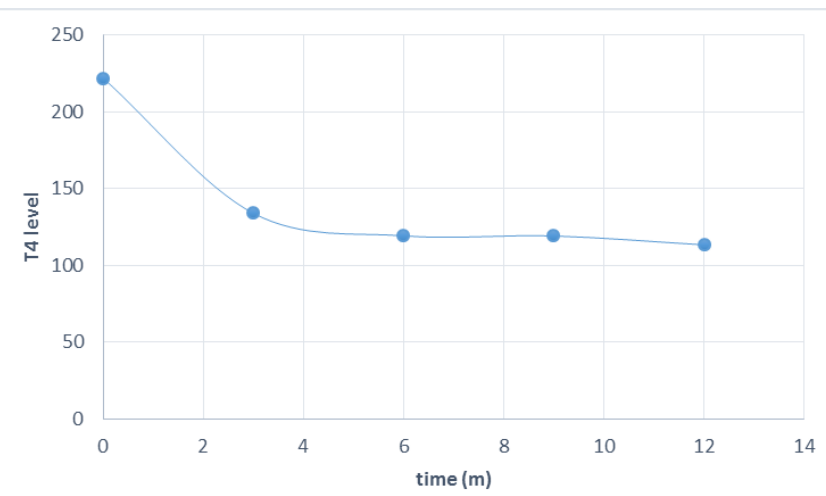

Figure 2: Line graph showed the correlation of T4 value with period of time in months

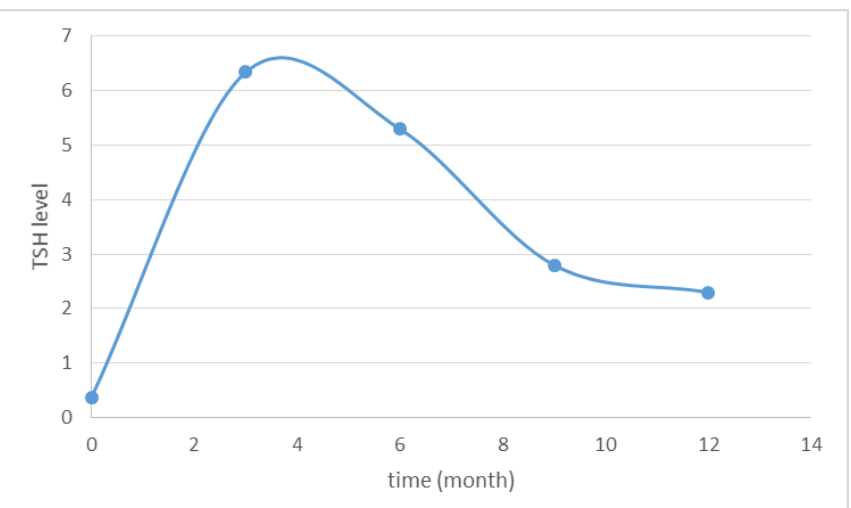

Figure 3: simple line polt demonstrate the recovery of TSH value after successful $\mathrm{I}^{131}$ ablation then decreased to reach normal level (nmol/L)

\section{Discussion}

In 1941 Radio-iodine treatment of thyroid gland had been proposed as a modality of choice and has since evolved to the treatment modality of choice for the majority of adult patients particularly in USA, UK and Europe, while the drugs (antithyroid drugs) can cause complete remission in $10-40 \%$ of patient only, William H. et.al 2005. Administered dose is usually calculated with the goal of administering approximately 70-120 Gy (7000-12000 rad) to the thyroid gland [4]. The calculation is made as follows: administered microcuries $=\mu \mathrm{Ci} / \mathrm{g}$ desired $\times$ gland weight $(\mathrm{g}) \times 100 \div$ percent uptake (24 hours). This equation demonstrates the effect of thyroid uptake in calculation of administered dose.

A mean dose used to ablate thyroid tissue was $(13.45 \mathrm{mCi})$. This was be decided upon (1) the amount of radioiodine administered, (2) the fraction deposited in the gland (uptake), (3) the duration of retention by the thyroid (biologic halflife), and (4) the radio sensitivity of the irradiated tissue.

The main purpose of this study is to assess the level of thyroid hormones in patients with hyperthyroidism in five periods which are (before treatment, 3 months after treatment, 6months, 9 months and 12 months after treatment) for T3, T4 and level of TSH. The result showed that the mean value of these hormone was $6.9,4.2,2.8$, and $2.5(\mathrm{nmol} / \mathrm{L})$ for $\mathrm{T} 3$, 222.1, 134, 119, 119.2 and $113.4(\mathrm{nmol} / \mathrm{L})$ for T4, and 0.37 , $6.34,5.3,2.8$, and $2.3(\mathrm{nmol} / \mathrm{L})$ for TSH respectively, table
(1). These were compared to the normal level of thyroid hormone which are T3 $(0.8-3 \mathrm{nmol} / \mathrm{L}), \mathrm{T} 4(50-150 \mathrm{nmol} / \mathrm{L})$ and TSH (0.4-4 nmol/L) and we noted an elevated thyroid hormones before treatment and then gradually decreased over the mentioned period of time for assessment this result reveals that the hormone level were decreased in response to the treatment.

A significant paired sample T-test was performed in order to test the difference between these values for the same patients in mentioned period. The result showed that there is a significant difference between the thyroid hormone level before $\mathrm{I}^{131}$ adminstration and after 3, 6, 9, and 12 months later after the treatment, at Confidence level of $(\mathrm{CL}=95 \%)$ and $(\mathrm{p}$ value $=0.05)$, the test were significant at $\mathrm{P}=0.000$, which mean that the thyroid hormone are significantly changing in these period which indicate the effectiveness of the I-131 to treat the hyperthyroidism and its causative factor such as TMNG, or Graves' disease.

Factor or disease that may lead to cause hyperthyroidism was also investigated and our result showed that 45 patient (54\%) of the collected samples were caused by Graves' disease, 28 $(23.3 \%)$ by TMNG, 30 (25\%) due to Diffuse toxic goiter and $6.7 \%$ related to the autonomous nodules. William $\mathrm{H}$. et.al 2005 stated that $95 \%$ of hyperthyroidism caused by Graves' disease and diffuse toxic goiter, as in table (3).

Figure (1): showed that linear relationship between the T3 level and the time before and after the treatment which demonstrated the decrement of $\mathrm{T} 3$ value with period of time in which decreased by $0.3455(\mathrm{nmol} / \mathrm{L})$ for every one month increment in time after treatment by I-131 $(y=-\mathbf{0 . 3 4 5 5 x}+$ 5.8996, $\boldsymbol{R}^{2}=\mathbf{0 . 7 7 3 6}$ ). Assessment of T3, T4, and TSH value after 6 month is consider significant in term of treatment response as stated by William H et.al 2005.

Figure (2): showed strong correlation between T4 value and the time in similar manner as $\mathrm{T} 3$, that result may explained by physiological aspect of $\mathrm{T} 4$ value which is derivative from the T3 as normal physiological process, so when level of T3 decreased T4 is decreased due to lack of T3 synthesized by thyroid tissue related to normal iodine uptake. The correlation showed that T4 level decreased by 7.746 $(\mathrm{nmol} / \mathrm{L})$ every month increments in period after thyroid treatment. $y=-7.746 x+188.05, R^{2}=0.6472$.

Figure (3): demonstrate the effect of treatment on the level of TSH after the treatment, in normal pathophysiological process of hyperthyroidism TSH level where decreased, due to overexpression of T3 and T4 in the blood, as stated by A. zeiessman et.al 2006. our study note the same result in which TSH having value of $0.37(\mathrm{nmol} / \mathrm{L})$ then starting to increase until starting the treatment, reaching a value of $6.34(\mathrm{nmol} / \mathrm{L})$ in three month (peak value), then starting to decrease as a function of time (month) indicating of hypothyroidism, in which decreased by $0.434(\mathrm{nmol} / \mathrm{L})$ for every one month increment in period after I131 administration. $\boldsymbol{y}=$ $0.4347 x+7.3437 R^{2}=0.9446$. This result was explained by William $\mathrm{H}$ et.al 2005, who stated that following radioiodine

\section{Volume 4 Issue 12, December 2015}




\section{International Journal of Science and Research (IJSR) \\ ISSN (Online): 2319-7064 \\ Index Copernicus Value (2013): 6.14 | Impact Factor (2013): 4.438}

therapy, the patient should be advised to have serum thyroid hormone and TSH levels checked within 2 to 3 months. Patients may be symptomatically improved within 4 to 6 weeks, but clinically significant hypothyroidism rarely occurs before 2 to 3 months. Hypothyroidism is only a problem if not adequately treated, and many practitioners will initiate thyroxine replacement therapy at the earliest indication of post-therapy hypothyroidism.

\section{Conclusion}

Radio-iodine treatment is considered one of the effective method used in treatment of thyroid disease in nuclear medicine departments, this study revealed that there is a significant difference in thyroid hormone level before and after treatment in which the effectiveness of the treatment were assessed.

\section{References}

[1] Abdallah YMY. Wagiallah EW. 2014. Enhancement of Nuclear Medicine Images using Filtering Technique, International Journal of Science and Research. Volume 3, Issue 8, pp. 916-921

[2] Wagiallah EW. Ahmed Y. Abdallah YMY.2014. Correction Preprocessing Method for Cardiac Scintography Images using Local Adaptive Filters, International Journal of Science and Research. Volume 3, Issue 8, pp. 1885-1889

[3] Abdallah YMY. Abdelwahab RI. 2014. Application of Texture Analysis Algorithm for Data Extraction in Dental X-Ray Images, International Journal of Science and Research. Volume 3, Issue 8, pp.1934-1937

[4] Adam MJ, Wilbur DS .2005. Radiohalogens for imaging and therapy. Chem Soc Rev 34:153-63

[5] Adelson, E.H., Bergen, J.R. 1991. "The plenoptic function and the elements of early vision", In Computation Models of Visual Processing, M. Landyand J.A. Movshon, eds., MIT Press, Cambridge, 1991, pp. 320.

[6] Arvo, J., 1994, The Irradiance Jacobian for Partially Occluded Polyhedral Sources, Proc. ACM SIGGRAPH, ACM Press, pp. 335-342.

[7] Ball, J., Moore, A., 1997, Essential physics for radiographers, 3rd edition, Blackwell Scientific, Oxford.

[8] Ball, J., Price, T., 1995, Chesney's radiographic imaging, 6th edition, Blackwell Scientific, Oxford.

[9] Buehler, C., Bosse, M., McMillan, L., Gortler, S., Cohen, M., 2001, Unstructured Lumigraph rendering, Proc. ACM SIGGRAPH, ACM Press.

[10]Farr, R., Allisy-Roberts, P., 1997, Physics for medical imaging, W.B. Saunders, London. Kusic Z, Becker DV, Saenger EL, et al. Comparison of technetium-99m and iodine123 imaging of thyroid nodules: correlation with pathologic findings. J Nucl Med 1990; 31:393-399.

[11] Mazzaferri EL. Management of a solitary thyroid nodule. N Engl J Med 1993; 328:553-559.

[12] Shakir F, Fitzsimmons TR, Jaques DP, et al. Diagnosis and management of the autonomously functioning thyroid nodule: theWalter Reed ArmyMedical Center experience, 1975-1996. Thyroid 1998; 8:871-880.
[13] Graham GD, Burman KD. Radioiodine treatment of Graves' disease. An assessment of its potential risks. Ann Intern Med 1986; 105:900-905.

\section{Author Profile}

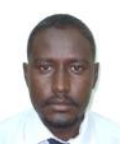

Mr. Mahdi Ahmed Ibrahim (Sudan) received the B.Sc. in radiotherapy technology from College of Medical radiological Science, Sudan University of Science and Technology in 2003, during 2003and up to 2014. He had been working at Nuclear medicine department, radiation and isotopes center of Khartoum, during 2014 up to date he has been working as NM practitioner at nuclear medicine department at Sabah Al-Ahmed Urology Center (SAUC), Kuwait.

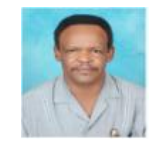

Assoc. Proff. Mohamed Elfadil Mohamed Garelnabi (Sudan Medicine (1987) and M.Sc. in Radiation Therapy) awarded the B.Sc. in Radiotherapy and Nuclear (2000-SUST) and Ph. D. degree in Medical Physics (Natal University-South Africa) in 2007. During 1996-2012 he has been working as lecturer as well as Associate Prof. at SUST department of Radiation therapy. Also he has been active in Computerized Texture Analysis, Radiotherapy- Oncology, Ultrasound and Nuclear Medicine researches.

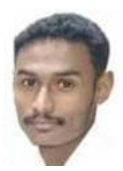

Abdoelrahman Hassan Ali Bakry (Sudan) received the B.Sc. and M.Sc. in radiotherapy technology from College of Medical radiological Science, Sudan University of Science and Technology in 2013. During 2013 up to date, he is staying in College of Medical radiological Science, Sudan University of Science and Technology, Radiology Department, Antalya Medical Center and Elnileen Diagnostic Medical Center; also he has been active in Computerized Texture Analysis, Radiotherapy-Oncology, and diagnostic radiology and Nuclear Medicine researches. Now he is teaching assistant at SUST also (2014). 